\title{
Catechol-O-methyltransferase polymorphism modulates cognitive control in children with chromosome 22q11.2 deletion syndrome
}

\author{
Yukari TAKarae, Linda Schmidt, Flora Tassone, ANd Tony J. Simon \\ University of California, Davis, California
}

\begin{abstract}
Dopamine plays a critical role in regulating neural activity in prefrontal cortex (PFC) and modulates cognition via a hypothesized inverse $U$ function. We investigated PFC function in children with chromosome 22q11.2 deletion syndrome (22q11.2DS) in which one copy of catechol-O-methyltransferase (COMT) is deleted, thereby shifting them toward the lower end of dopamine turnover on the nonlinear function. A common polymorphism with valine to methionine substitution alters COMT activity that results in higher enzyme activity in the valine variant. Twenty-seven children with 22q11.2DS between 7 and 14 years old, and 21 age-matched typically developing children, performed a modified version of the Attention Network Test. Children with a single valine allele showed a reduction in response times when trials with incongruent flankers were repeated, whereas those who were hemizygous for the methionine allele did not show the same context-based response facilitation. Our results support that a single gene, COMT, could modulate PFC-dependent cognition.
\end{abstract}

Chromosome 22q11.2 deletion syndrome (22q11.2DS) results from a $1.5-$ to $3.0-\mathrm{Mb}$ deletion on the long arm of chromosome 22 at location q11.2 (Maynard, Haskell, Lieberman, \& LaMantia, 2002). Affected individuals typically have full-scale IQ scores of 70-85 with significant impairments in spatial attention, numerical cognition (Simon, Bearden, McGinn, \& Zackai, 2005), and executive function (Bish, Ferrante, McDonald-McGinn, Zackai, \& Simon, 2005; Sobin, Kiley-Brabeck, \& Karayiorgou, 2005). The q11.2 region of chromosome 22 includes the catechol-O-methyltransferase (COMT) gene, which is critical in metabolizing dopamine by methylation. A common polymorphism involving a valine (VAL) to methionine (MET) substitution at codon 158 in the aminoacid sequence changes the stability of enzyme activity. As a result, the VAL variant produces $40 \%$ more enzyme activity than does the MET variant at normal body temperature (Chen et al., 2004). Because the dopamine transporter is much less abundant in prefrontal cortex (PFC) than in the striatum, the synaptic deactivation of dopamine in PFC depends to a much greater degree on COMT. For example, methylation accounts for more than $60 \%$ of metabolic degradation of dopamine in frontal cortex in comparison with $15 \%$ in the striatum and nucleus accumbens (Karoum, Chrapusta, \& Egan, 1994).

The observation that variation in a single gene could explain a large amount of variance in dopamine metabolism in PFC is particularly interesting in light of previous reports of a mediating role for dopamine in higher cognitive functions (Kimberg \& D'Esposito, 2003). Although it is difficult to accurately measure dopamine levels in the human brain, genotypes for COMT could be used as an indicator of dopamine metabolism. The efficiency of PFC-mediated cognition in typically developing (TD) adults has already been shown to vary by the COMT genotype. A large-sample study by Egan et al. (2001) demonstrated that TD adults with homozygous VAL alleles made more perseverative errors than did those with homozygous MET alleles during the Wisconsin Card Sort Task, and this effect was deemed reliable in a more recent meta-analysis based on 17 published studies (Barnett, Jones, Robbins, \& Müller, 2007). Egan et al. also conducted a functional imaging study using an $N$-back task in which participants were asked to indicate whether the current stimulus was the same as the one presented $N$ trials before. They found that those with homozygous VAL alleles produced greater activation in PFC and in the anterior cingulate - which also receives rich dopaminergic innervation - than did those with homozygous MET alleles. The observed difference in brain activation when the performance level of the two groups was similar suggests less efficient processing in the relevant PFC circuitry in the VAL group. Similar findings were found with different cognitive paradigms that required high demands for cognitive control and attention regulation (Blasi et al., 2005; Winterer et al., 2006).

It has been shown that the effect of dopamine levels on cognition follows an inverse $U$ function in which a relatively small range of dopamine optimizes cognitive performance (Williams \& Goldman-Rakic, 1995). Because 
they are missing one copy of the COMT gene, individuals with 22q11.2DS are considered to have lower levels of dopamine turnover than do TD individuals (Boot et al., 2008). Effects of dopamine-related genes on dopamine transmission is additive (Bertolino et al., 2006), and VAL alleles produce $40 \%$ higher enzyme activity at normal body temperature (Chen et al., 2004). Thus, if enzyme activity associated with MET alleles is defined as having the value 1 , and one associated with VAL alleles is defined as having the value 1.4, then expected COMT activity levels will be highest with the Val/Val (2.8) genotype, followed by Val/Met (2.4), Met/Met (2.0), hemizygous Val (1.4), then hemizygous Met (1.0) genotypes. Given their hemizygous status for the COMT gene, individuals with 22q11.2DS would thus be expected to manifest lower enzyme levels than would TD individuals, with those hemizygous for the MET allele situated at a more extreme position on the inverse $U$ function than those hemizygous for the VAL allele (Gothelf et al., 2007). Consistently, cognitive processes that are associated with PFC are frequently impaired in the 22q11.2DS population. For example, poor prepulse inhibition, which is the suppression of startle responses that follows warning cues and which is modulated by dopamine in the orbitofrontal cortex (Zavitsanou, Cranney, \& Richardson, 1999), has been documented in 22q11.2DS (Sobin et al., 2005).

Although individuals with 22q11.2DS represent a different range on the inverse $U$ function than do TD individuals, their cognitive performance - albeit inconsistentlyseems to also vary as a function of COMT genotype. Some studies have reported superior performance in those with hemizygous MET alleles on the continuous performance task (Shashi et al., 2006), the Wisconsin Card Sort Task (Kates et al., 2006), and in composite scores from Arithmetic, Digit Span from WISC-III, verbal fluency, and Trail B tests (Bearden et al., 2004). Others have reported no difference by COMT genotype (Glaser et al., 2006) or superior performance on a working memory task (Baker, Baldeweg, Sivagnanasundaram, Scambler, $\&$ Skuse, 2005) or greater PFC volume (Gothelf et al., 2005 ) in those with hemizygous VAL alleles. These differences are difficult to interpret because of differences in participants' ages and tasks employed. Diamond, Briand, Fossella, and Gehlbach (2004), for instance, reported that COMT genotypes could predict cognitive performance on the task that requires inhibiting prepotent responses, but not on other tasks that depend on PFC but that do not require such executive control of behaviors.

We (Bish et al., 2005) have previously reported impairments in PFC function in children with 22q11.2DS using a modified version of the attention network task (ANT) (Fan, McCandliss, Sommer, Raz, \& Posner, 2002). Among other things, the ANT assesses the executive component of attention using flankers that are congruent or incongruent with the target stimuli, which requires participants to inhibit responses to these flankers and selectively focus on the target. TD children showed improvements in performance when they were presented with the same type of flankers on successive trials. This is referred to as conflict adaptation, or the Gratton effect (Gratton, Coles, \& Donchin, 1992). As a group, children with 22q11.2DS, however, failed to show the same type of response facilitation based on the executive control using prior trial context when the target stimulus was presented with incongruent flankers. Because individuals with 22q11.2DS are missing one copy of the COMT gene that affects dopamine turnover in PFC, the present study evaluated the hypothesis that our previous findings on the Gratton effect could be modulated by COMT genotype. More specifically, because those with a single MET allele are positioned at most extreme positions on the inverse $U$ function, we expected those with a single MET allele to perform worse than those with a single VAL allele.

\section{METHOD}

\section{Participants}

A total of 48 children between 7 and 14 years of age participated in the present study. After a complete description of the study, written consent and/or assent was given by all participants or their parents. The protocol was approved by the Institutional Review Board at the University of California, Davis. None of the participants was administrated ANT in our previous study (Bish et al., 2005). Twenty-seven children were diagnosed with 22q11.2DS via standard fluorescence in situ hybridization (FISH) testing. The remaining 21 participants were TD children. Of 27 children with 22q11.2DS, 16 were hemizygous for the MET allele, and 11 were hemizygous for the VAL allele. Since only 8 TD children volunteered to submit biological samples for genotyping, the sample was too small to allow comparisons across genotypes ( $n=2$ for homozygous MET, $n=3$ for homozygous VAL, and $n=3$ for heterozygous alleles). Thus, no analysis by genotype was performed with TD children. Mean ages for each group were $130.84(S D=22.05)$ months for the hemizygous MET group, $132.00(S D=22.33)$ months for the hemizygous VAL group, and $130.05(S D=24.90)$ months for the TD group. Percentages of male and female participants, respectively, were $43.8 \%$ and $56.3 \%$, for the hemizygous MET group, $54.5 \%$ and $45.5 \%$ for the hemizygous VAL group, and $52.4 \%$ and $47.6 \%$ for the TD group. Neither chronological age nor sex ratios were statistically different between groups. Intelligence quotients from the Wechsler Intelligence Scale for Children-IV were available for 23 participants with 22q11.2DS and 13 TD children. Two 22q11.2DS groups were matched on full-scale IQ, verbal comprehension, perceptual reasoning, and processing speed index scores, although both 22q11.2DS groups statistically differed from the TD group on these measures (Table 1).

\section{Molecular Analyses}

Genomic DNA was isolated from peripheral blood leukocytes and saliva samples by standard methods (Puregene, Gentra, MN; Oragene; DNA genoteck, Ottawa, Ontario, Canada). Genotyping analysis for the COMT Val ${ }^{108 / 158}$ Met was carried out by PCR analysis, followed by restriction enzyme digestion. Cycling conditions were $94^{\circ} \mathrm{C}$ for $3 \mathrm{~min} ; 15$ cycles of $94^{\circ} \mathrm{C}$ for $30 \mathrm{sec}, 68^{\circ} \mathrm{C}$ (minus $1^{\circ} \mathrm{C}$ per cycle) for $45 \mathrm{sec}, 72^{\circ} \mathrm{C}$ for $30 \mathrm{sec} ; 20$ cycles of $94^{\circ} \mathrm{C}$ for $30 \mathrm{sec}$, $53^{\circ} \mathrm{C}$ for $45 \mathrm{sec}, 72^{\circ} \mathrm{C}$ for $30 \mathrm{sec}$; and $72^{\circ} \mathrm{C}$ for $7 \mathrm{~min}$. A nested PCR, generating a 188 -bp fragment, was amplified in a 50- $\mu 1$ reaction containing standard reagents and $1 \mu \mathrm{l}$ of the first PCR reaction. $\mathrm{Cy}-$ cling conditions were $95^{\circ} \mathrm{C}$ for $5 \mathrm{~min} ; 35$ cycles of $95^{\circ} \mathrm{C}$ for $30 \mathrm{sec}$, $62^{\circ} \mathrm{C}$ for $30 \mathrm{sec}, 72^{\circ} \mathrm{C}$ for $30 \mathrm{sec}$; and $72^{\circ} \mathrm{C}$ for $2 \mathrm{~min}$. The products were visualized on a $2 \%$ agarose gel. Restriction enzyme digestion of the 188-bp product was performed in a $25-\mu 1$ reaction volume containing 15 units of Nla III (New England BioLabs) that was incubated overnight at $37^{\circ} \mathrm{C}$. Fragments were visualized on a Novex 4\%-20\% TBE gel (Invitrogen). 
Table 1

Performance on the Wechsler Intelligence Scale for Children-IV

\begin{tabular}{|c|c|c|c|c|c|c|c|c|}
\hline & \multicolumn{2}{|c|}{$\begin{array}{c}\text { Typically } \\
\text { Developing } \\
\text { (TD) }\end{array}$} & \multicolumn{2}{|c|}{$\begin{array}{c}\text { 22q11.2DS } \\
\text { Hemizygous } \\
\text { MET }\end{array}$} & \multicolumn{2}{|c|}{$\begin{array}{c}\text { 22q11.2DS } \\
\text { Hemizygous } \\
\text { VAL }\end{array}$} & \multirow[b]{2}{*}{$F(2,32)$} & \multirow[b]{2}{*}{$p<$} \\
\hline & $M$ & $S D$ & $M$ & $S D$ & $M$ & $S D$ & & \\
\hline Full-scale IQ & 112.15 & 8.97 & $78.86^{* * *}$ & 14.65 & $79.00^{* * *}$ & 7.69 & 34.67 & .001 \\
\hline Verbal comprehension index & 113.08 & 9.45 & $79.64^{* * *}$ & 13.87 & $80.25^{* * *}$ & 5.34 & 38.27 & .001 \\
\hline Perceptual reasoning index & 114.23 & 8.60 & $80.07^{* * *}$ & 15.71 & $86.50^{* *}$ & 13.21 & 25.54 & .001 \\
\hline Processing speed & 100.46 & 17.59 & $78.13^{*}$ & 5.17 & $86.36^{* *}$ & 11.11 & 7.95 & .01 \\
\hline
\end{tabular}

Note-Both 22q11.2DS groups differed from the control group on full-scale IQ scores, verbal comprehension, perceptual reasoning, and processing speed index scores, although they did not statistically differ from each other. $F$ statistics from one-way ANOVAs comparing three groups and $p$ values from post hoc Bonferroni tests comparing each of the two 22q11.2DS groups and the TD group are presented. ${ }^{*} p<.06$ when compared with the TD group. ${ }^{* *} p<.01$ when compared with the TD group. ${ }^{* * *} p<.001$ when compared with the TD group.

\section{Task Procedures}

A modified version of ANT was used to assess the Gratton effect. Detailed descriptions of the task have been published elsewhere (Bish et al., 2005). Briefly, the child was asked to identify the direction of an arrow drawn on a friendly alien spaceship figure (approximately $1.5^{\circ}$ wide) appearing on a computer monitor. The target spaceship appeared approximately $1.9^{\circ}$ of visual angle above or below a central fixation point. The target appeared at these locations at equal probabilities, and the child was asked to indicate the direction of the arrow by buttonpress. The target spaceship was presented in the context of four spatial cuing conditions in which one of the following cue types preceded its appearance: none (no cue presented), valid (cue appears in the same location as the target), neutral (cue appears at center and thus provides no useful spatial information), or invalid (cue appears in the opposite location from the target). The valid cue condition occurred on $75 \%$ of trials with a spatially useful cue (i.e., valid or invalid, but not neutral). All cue types were collapsed for the present analysis because the effect of spatial cues was not the focus of this investigation.

The target was presented with or without flankers, and the child was instructed to ignore the flankers. The flankers were spaceships identical to the target stimulus, and two flankers were presented on each side of the target stimulus. The direction in which the arrows on the flanker spaceships pointed was either the same as (congruent) or the opposite of (incongruent) that of the target arrow (Figure 1). Three flanker conditions (none, congruent, or incongruent) were equally distributed across all cue types. In order to examine the Gratton effect, trials with congruent or incongruent flankers were classified on the basis of how many congruent or incongruent trials preceded the current trial. One difference from our previous version of this task was a new manipulation of the sequence of trials that was designed to create a pseudorandom set within which we could present the same flanker type three-rather than two-times in a row. This manipulation was added to assess whether adaptation - as is defined by the Gratton effect - was not possible for some participants to achieve, or whether it was merely impaired to the extent that a second repeat of the same event was required. Trialwise response times (RTs) were excluded at each participant level from the analysis if they were more than 2.5 standard deviations from the grand mean. Median RTs were computed for each trial category.

In addition to ANT, simple reaction time was measured in order to assess basic psychomotor speed. The task consisted of a friendly alien figure (approximately $4.1^{\circ}$ high $\times 1.4^{\circ}$ wide) appearing with unpredictable timing at either the right or the left side of the entrance (approximately $4.1^{\circ}$ high $\times 2.4^{\circ}$ wide) of a house that was presented on the computer monitor. On each trial, the child's task was to indicate, by pressing a single button as quickly as possible, the appearance of the alien, which appeared with variable delay $(400,800$, or $1,200 \mathrm{msec}-20$ trials of each) in order to minimize anticipatory responses. The median RTs across all trials and all delays were used for statistical analysis.

\section{Statistical Analyses}

RTs from the simple reaction time task were analyzed using a one-way ANOVA that compared hemizygous MET, hemizygous VAL, and TD groups. RTs from ANT were analyzed using group $\times$ number of flanker repetition ANOVAs for congruent and incongruent trials. Degrees of freedom were adjusted using the Huynh-Feldt method to correct for possible violations of the sphericity assumption. This was followed by trend analyses because our primary interest was to examine whether participants showed systematic reduction in RT to reflect conflict adaptation during this task when the same flanker types were repeated. The degree to which this RT reduction is consistently observed in our participant groups should be reflected in the strength of the linear trend in RT changes. Although the trend analysis examined patterns of RT changes at group levels, we also computed slopes of RT changes within each participant to examine the degree of conflict adaptation at individual levels. When statistically significant group differences were identified, the difference was followed up by either $t$ tests or linear contrasts, which allowed both simple and complex group comparisons, with Bonferroni correction.

To examine potential changes in speed-accuracy trade-off during cognitive assessment, we also examined error rates during the ANT task. Because of nonnormal distributions of error rates, we performed square root transformation on error rates. Then, trans-
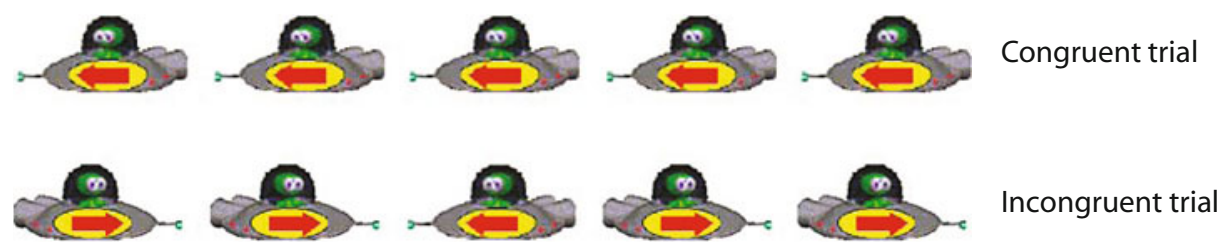

Figure 1. Example of stimuli used in the present study. Participants were asked to indicate the direction of center arrows. Congruent and incongruent trials are shown. 


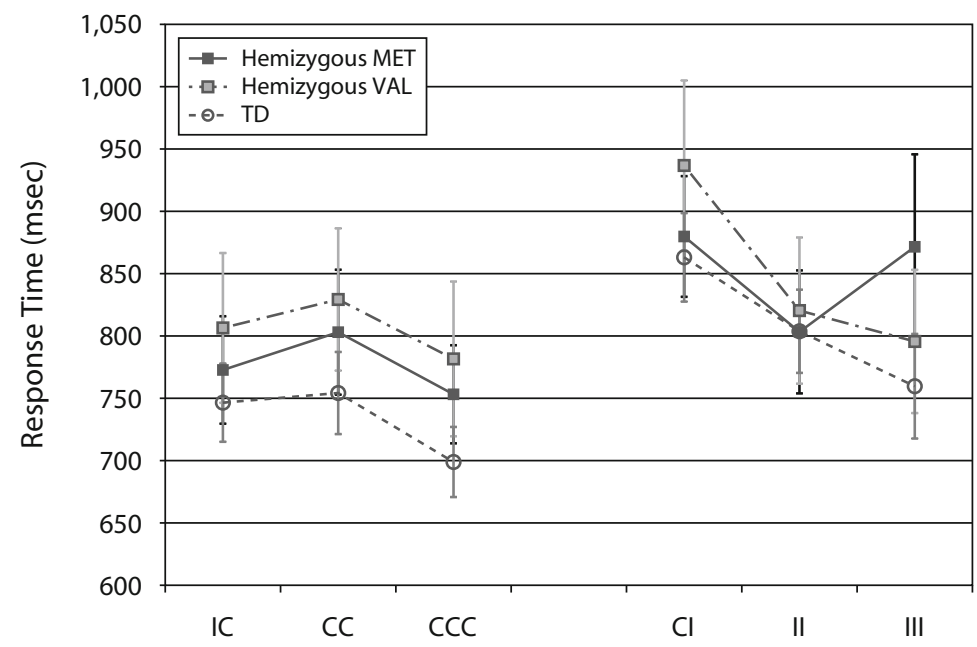

Figure 2. Mean response time by trial type. The $x$-axis shows how many times the current congruent or incongruent trial was preceded by the same flanker type. IC, congruent preceded by incongruent trial; CC, congruent preceded by single congruent trial; $\mathrm{CCC}$, congruent preceded by two successive congruent trials; CI, incongruent preceded by congruent trial; II, incongruent preceded by single incongruent trial; III, incongruent preceded by two successive incongruent trials.

formed scores were submitted to an ANOVA and trend analysis to conduct parallel analyses with RT data. We further computed slopes for error rate changes across conditions in each individual participant and examined group differences in the slopes.

\section{RESULTS}

There were no group differences in basic psychomotor speed as measured by the simple reaction time task $[F(2,40)=1.52$, n.s. $](M=371.05 \mathrm{msec}, S D=105.84$, for MET; $M=310.87 \mathrm{msec}, S D=77.16$, for VAL; $M=$ $370.04 \mathrm{msec}, S D=83.57$, for TD). Therefore, we assume that all significant differences in RTs obtained from ANT described below were produced by differences in cognitive processing. A group $\times$ number of flanker repetition ANOVA for congruent trials revealed no significant differences by group or a group $\times$ flanker repetition interaction $(F \mathrm{~s}<1)$. The same ANOVA for incongruent trials showed no significant group difference $(F<1)$, but there was a significant group $\times$ flanker repetition interaction $[F(4,90)=2.748, p<.05]$. This significant interaction appears to be related to the observation that, at the group level, both TD children and children hemizygous for the VAL allele showed consistent reduction in RT when incongruent trials were repeated, whereas children hemizygous for the MET allele did not show consistent reduction in RT (Figure 2). None of the pairwise comparisons resulted in statistically significant differences except for changes from single incongruent trials $(\mathrm{CI})$ to two successive incongruent trials (II) $[t(15)=3.45, p<.05$, for the hemizygous MET group; $t(10)=4.51, p<.01$, for the hemizygous VAL group; and $t(20)=3.57, p<.01$, for the TD group]. The group $\times$ flanker repetition interaction was also followed up by analyses of polynomial trends. Reflecting the systematic reduction in RT with flanker repetition, significant linear trends were observed for hemizygous VAL and TD groups for incongruent trials, but not for the hemizygous MET group $[F(1,10)=26.41, p<.001$, for the hemizygous VAL group; $F(1,20)=15.94, p<.01$, for the TD group; $F<1$ for the hemizygous MET group]. The MET group, however, showed a significant quadratic trend $[F(1,15)=$ $6.45, p<.05]$. During the congruent trials, only the TD group showed RT reduction with a significant linear trend $[F(1,20)=4.50, p<.05]$.

In order to examine the possibility that RT changes reflect a speed-accuracy trade-off, a group $\times$ flanker repetition ANOVA was run with transformed error rates (Figure 3). There was no significant group difference or any group $\times$ flanker repetition interaction in the congruent condition $(F \mathrm{~S}<1)$ or in the incongruent condition $[F(2,45)=1.83$, n.s., for group; $F<1$ for group $\times$ flanker repetition]. We modeled changes in error rates at group levels by examining polynomial trends, as we did with RT. There were no significant linear or quadratic trends in any groups in either congruent or incongruent conditions [congruent condition, $F(1,15)=1.96$, n.s., for linear, and $F<1$ for quadratic trends for the MET group; $F \mathrm{~s}<1$ for both trends for the VAL and TD groups; incongruent condition, $F(1,15)=2.83$, n.s., for linear, and $F<1$ for quadratic trends for the MET group; $F<1$ for linear, and $F(1,10)=2.22$, n.s., for quadratic trends for the VAL group; $F_{\mathrm{S}}<1$ for both trends for the TD group].

To examine whether the performance patterns observed at group levels were found at the level of individual participants, RT changes for each participant were characterized by computing slopes of best-fitted linear function for RT changes. For congruent trials, average slopes for each group were $-9.77(S D=60.22)$ for the hemizygous MET group, $-12.43(S D=42.17)$ for the hemizygous VAL group, and $-23.81(S D=51.43)$ for the TD group. For incon- 


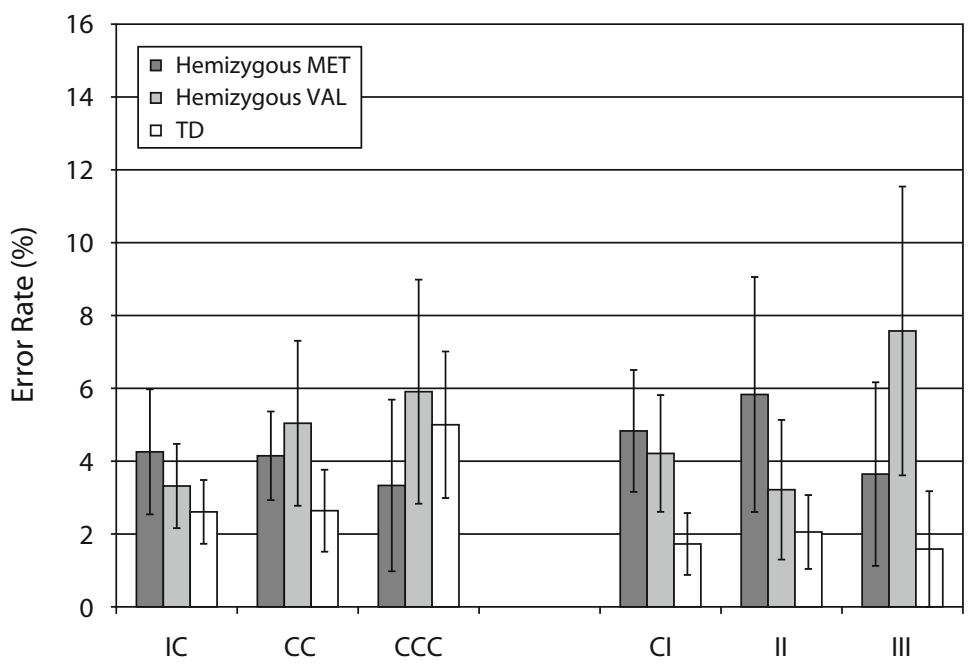

Figure 3. Raw error rates by trial type. The $x$-axis shows how many times the current congruent or incongruent trial was preceded by the same flanker type. IC, congruent preceded by incongruent trial; $\mathrm{CC}$, congruent preceded by single congruent trial; $\mathrm{CCC}$, congruent preceded by two successive congruent trials; CI, incongruent preceded by congruent trial; II, incongruent preceded by single incongruent trial; III, incongruent preceded by two successive incongruent trials.

gruent trials, average slopes were $-4.14(S D=100.38)$ for the hemizygous MET group, $-70.59(S D=45.55)$ for the hemizygous VAL group, and $-51.70(S D=59.34)$ for the TD group. Only the slopes from incongruent trials were statistically different between groups $[F(2,45)=$ $3.13, p=.053]$. Post hoc linear contrast tests demonstrated that slopes from children in the hemizygous MET group were smaller than those from the hemizygous VAL group $[t=2.31, p<.05]$ or from both the VAL and TD groups combined $[t=2.49, p<.05]$. Cohen's $d$ comparing slopes in the two 22q11.2DS groups was 0.05 for congruent and 0.80 for incongruent trials. Furthermore, COMT genotype accounted for $0.06 \%$ and $14.36 \%$ of the variance in slopes for congruent and incongruent trials, respectively, in the 22q11.2DS group. We also computed slopes of error rate changes for each individual participant. Mean slopes for congruent and incongruent conditions were $-0.46 \%$ ( $S D=$ $3.45)$ and $-0.59 \%(S D=4.34)$ for the MET group, $1.29 \%$ $(S D=5.67)$ and $1.68 \%(S D=5.89)$ for the VAL group, and $1.19 \%(4.67)$ and $-0.06 \%(S D=4.29)$ for the TD group. A one-way ANOVA revealed no significant group differences in slopes of error rates in either the congruent or incongruent condition $(F \mathrm{~s}<1)$.

Although all participants who were hemizygous for the VAL allele had negative slopes from incongruent trials (suggesting that they all showed some degrees of conflict adaptation), a substantial portion of participants who were hemizygous for the MET allele had near-zero or positive slopes that suggested abnormal conflict adaptation (Figure 4). All slope values from the VAL group were less than $-20 \mathrm{msec}$, and $62.5 \%$ of participants from the MET group had slopes in the same range. Thus, $37.5 \%$ of participants who were hemizygous for the MET allele had slope values that were larger than those of any participant with hemizygous VAL alleles. Using a cutoff value of $-20 \mathrm{msec}$, participants with 22q11.2DS were classified into two groups, depending on whether they had slopes smaller (more consistent conflict adaptation) or larger (less consistent or abnormal adaptation) than the cutoff. A $\chi^{2}$ test showed that percentages of participants who had more consistent conflict adaptation were statistically different between the VAL and MET groups $\left[\chi^{2}(1)=5.30\right.$, $p<.05]$. Within the MET group, these participants who had slopes larger than -20 msec (less conflict adaptation) had overall longer RTs $(M=1,023.19 \mathrm{msec})$ and similar or higher error rates $(4.2 \%, 12.6 \%$, and $5.6 \%$ for CI, II, and III conditions, respectively) than did the remaining participants with the same genotype (RT mean, $748.53 \mathrm{msec}$; error rates, $5.2 \%, 1.7 \%$, and $2.5 \%$ for CI, II, and III conditions, respectively). Mean slopes for RT and error rates were $86.17 \mathrm{msec}(S D=100.02)$ and $0.69 \%(S D=6.67)$ for those who had slopes larger than $-20 \mathrm{msec}$, and $-58.33 \mathrm{msec}(S D=50.37)$ and $-1.36 \%$ $(S D=2.21)$ for those with smaller slopes. Although slopes for RTs were statistically different between these two subgroups with hemizygous MET alleles, slopes for error rates did not differ $[t(14)=3.297, p<.05$, for RTs and $t<1$ for error rates].

In order to examine whether the difference in slopes could be related to differences in more general psychomotor speed within the 22q11.2DS group, correlations between slopes and processing speed measures from the WISC-IV or RTs from the simple reaction time task were examined within the 22q11.2DS group. No significant correlations were found ( $r \mathrm{~s}-.233$ to .368 , n.s.); thus, the difference in the pattern of RT reduction in the two 22q11.2DS groups was not likely a simple function of psychomotor or cognitive speed. 


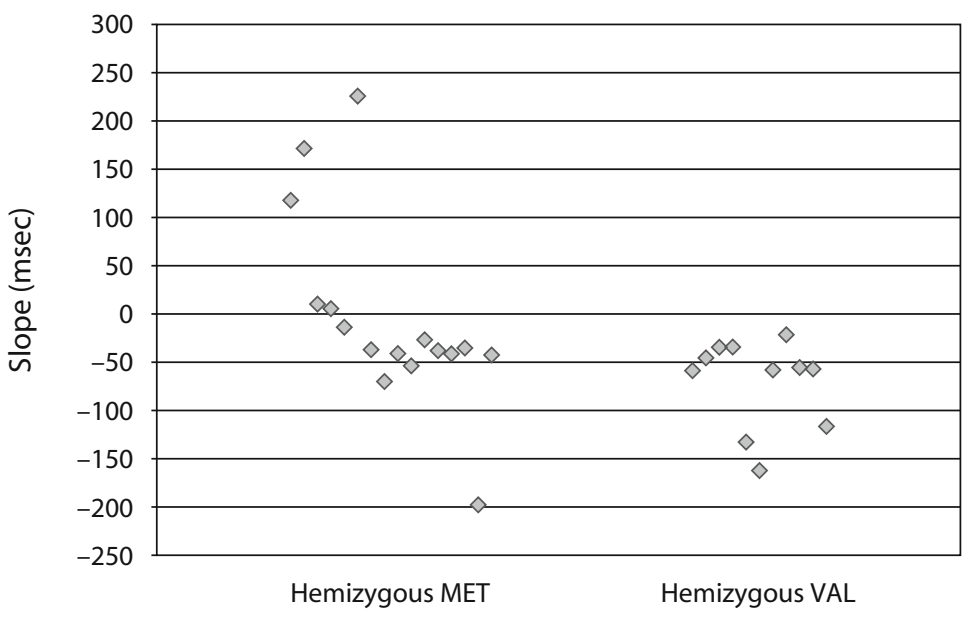

Figure 4. Individual slopes of response time changes from hemizygous MET and VAL groups from incongruent trials.

\section{DISCUSSION}

The present study was conducted to examine whether PFC-sensitive cognitive control, as measured by the Gratton effect during a flanker task, is modulated by COMT genotype in the 22q11.2DS population. Just like TD control children, those with 22q11.2DS who were hemizygous for the VAL allele were able to use prior trial context to help them adjust the amount of cognitive control required to overcome conflict presented in the form of incongruent flankers. This was observed in all participants with hemizygous VAL alleles. By contrast, a substantial portion of participants with hemizygous MET alleles failed to show consistent conflict adaptation. The observation that impairments in cognitive control were specific to those who were hemizygous for the MET allele suggests that the COMT genotype modulates conflict adaptation. However, impairments in conflict adaptation were not observed in all participants who were hemizygous for the MET allele; thus, there are some additional factors that are contributing to their difficulty with this specific type of cognitive control.

The Gratton effect that was examined in the present study reflects the ability to use prior task context to optimize behavioral responses in the context of needing to suppress responses to salient distractors and selectively attend to the target. It is a type of cognitive control that is critical in achieving goal-directed behaviors. Cognitive control of behaviors is supported by the network of brain areas, including the dorsolateral and ventrolateral prefrontal cortex, and the anterior cingulate cortex (Botvinick, Cohen, \& Carter, 2004; Bunge, Dudukovic, Thomason, Vaidya, $\&$ Gabrieli, 2002). The cortico-striato-thalamo-cortical loops are critical elements to support function of these brain areas, and they are regulated by complex interactions of glutamate, GABA, and dopamine systems (Grace, Floresco, Goto, \& Lodge, 2007; Honey et al., 2003). COMT significantly affects dopamine metabolism in PFC (Karoum et al., 1994) and consequently alters the function of the loop at multiple levels. PFC afferents to the stria- tum regulate activity of mesocortical dopamine neurons projecting to PFC via GABA neurons, which modulate working memory and executive function that are mediated by PFC (Seamans \& Yang, 2004; Winterer \& Weinberger, 2004). PFC afferents to the striatum are, in turn, regulated by midbrain dopamine systems as the PFC afferents and dopaminergic neurons terminate on single dendrites of striatal neurons (Freund, Powell, \& Smith, 1984; Kornhuber \& Kornhuber, 1983). The PFC-striato-thalamo-cortical loop receives regulatory input from the hippocampus that allows the binding of contextual information provided by the hippocampus with behavioral plans provided by PFC to update representations of the external world (Grace et al., 2007). Thus, dysregulation of this circuitry results in impairments in producing context-appropriate behaviors in order to achieve internally set goals.

It has been shown that the modulatory effect of dopamine on PFC function follows an inverse $U$ function at both synaptic and behavioral levels (Seamans \& Yang, 2004; Williams \& Goldman-Rakic, 1995). Although those with a single copy of MET or VAL allele are expected to have less COMT activity than would TD individuals, those with a single copy of MET allele are likely situated at more extreme points on the inverse $U$ function than are those with a single copy of VAL allele, because of the expected reduction in enzyme activity (Gothelf et al., 2005). Thus, it is likely that they deviate further from the optimal level for PFC function. This hypothesized position on the inverse $\mathrm{U}$ function is consistent with the present findings that children with hemizygous MET allele showed much less consistent conflict adaptation. It is, however, likely that the mechanism is more complex, since COMT genotypes could interact with other deleted or nondeleted genes that are involved in dopamine, glutamate, or GABA regulations, or possibly with atypical brain development, which could have cascading effects on neural systems connectivity and neurotransmitter regulation. For instance, atypical development of the limbic system has been observed in 22q11.2DS (Debbane, Schaer, 
Farhoumand, Glaser, \& Eliez, 2006; Eliez et al., 2001), whereas the limbic system provides regulatory input to the PFC-striato-thalamo-cortical loop.

Limitations of the study include a small sample size and the use of a flanker task that is sensitive to other cognitive processes in addition to conflict adaptation (Mayr, Awh, \& Laurey, 2003). Furthermore, because both dopaminergic innervations in the prefrontal cortex and COMT enzyme activity change with age (Rosenberg \& Lewis, 1995; Tunbridge et al., 2007), the present finding of cognitive control impairments in children with a single copy of the MET allele may be specific to the age group studied. Dopaminergic input to PFC reaches its peak in adolescence (Rosenberg \& Lewis, 1995), and COMT enzyme activity continues to increase until early adulthood (Tunbridge et al., 2007). Both of these could influence relative positions on the inverse $U$ function for each genotype-thus influencing cognitive performancethroughout development (Wahlstrom et al., 2007). Although it is not clear whether development of PFC in 22q11.2DS follows the same pattern as that observed in TD individuals, given high rates of schizophreniform disorders in this population (Murphy, 2002), past studies suggest that age could be one of the determining factors for how COMT genotypes influence dopamine levels and PFC function in 22q11.2DS. There seems to be a pattern that studies that used younger samples (Bearden et al., 2004; Kates et al., 2006; Shashi et al., 2006) (average ages of 9 to 11 years old) reported superior performance in those hemizygous for the MET allele, whereas studies that used older samples reported no difference or inferior performance in those hemizygous for the MET allele (Baker et al., 2005; Glaser et al., 2006; Gothelf et al., 2005) (average ages of 13 to 18 years old).

Although age might be one of the important factors to consider in our attempt to understand the development of PFC function in this population, part of the inconsistency in the literature might also be related to specific cognitive requirements for tasks used. Because PFC and dopamine systems are involved in many different cognitive processes in varying degrees, effects of COMT genes on cognition could vary in their directions and magnitude depending on specific task requirements for the study (Diamond et al., 2004). For instance, Bearden et al. (2004) reported superior performance in those who were hemizygous for the MET allele on tasks that have high loading on PFC, and the average age of their participants was similar to those in the present study. Their battery, consisting of Arithmetic, Digit Span from WISC-III, verbal fluency, and Trail B tests, however, does not have as high demands for behavioral inhibition and conflict monitoring as ANT that is employed in the present study. More studies are needed to clarify how cognitive impairments in 22q11.2DS are related to dopamine systems and brain development in this population.

\section{AUTHOR NOTE}

The present study was supported by NIH Grant R01-HD042974 to T.J.S. We thank Leeza Gabriel for her assistance in data collection, Joel
Bish for task development, and Carrie Bearden for comments on an earlier version of the manuscript. This work was presented in 2007 as a poster at the annual meeting of the Cognitive Neuroscience Society in New York. Address correspondence to Y. Takarae, University of California, Davis, Center for Mind and Brain, 276 Cousteau Place, Davis, CA 95618 (e-mail: ytakarae@ucdavis.edu).

\section{REFERENCES}

Baker, K., Baldeweg, T., Sivagnanasundaram, S., Scambler, P., \& SKUSE, D. (2005). COMT Val ${ }^{108 / 158}$ Met modifies mismatch negativity and cognitive function in 22q11 deletion syndrome. Biological Psychiatry, 58, 23-31.

Barnett, J. H., Jones, P. B., Robbins, T. W., \& Müller, U. (2007). Effects of the catechol-O-methyltransferase Val ${ }^{158}$ Met polymorphism on executive function: A meta-analysis of the Wisconsin Card Sort Test in schizophrenia and healthy controls. Molecular Psychiatry, 12, 502-509.

Bearden, C. E., Jawad, A. F., Lynch, D. R., Sokol, S., Kanes, S. J., McDonald-McGinn, D. M., ET AL. (2004). Effects of a functional COMT polymorphism on prefrontal cognitive function in patients with 22q11.2 deletion syndrome. American Journal of Psychiatry, 161, 1700-1702.

Bertolino, A., Blasi, G., Latorre, V., Rubino, V., Rampino, A., SinIBALDI, L., ET AL. (2006). Additive effects of genetic variation in dopamine regulating genes on working memory cortical activity in human brain. Journal of Neuroscience, 26, 3918-3922.

Bish, J. P., Ferrante, S. M., McDonald-McGinn, D., Zackai, E., \& Simon, T. J. (2005). Maladaptive conflict monitoring as evidence for executive dysfunction in children with chromosome 22q11.2 deletion syndrome. Developmental Science, 8, 36-43.

Blasi, G., Mattay, V. S., Bertolino, A., Elvevåg, B., Callicott, J. H., DAS, S., ET AL. (2005). Effect of catechol-O-methyltransferase val ${ }^{158}$ met genotype on attentional control. Journal of Neuroscience, 25, 5038-5045.

Boot, E., BooiJ, J., Zinkstok, J., Abeling, N., de Haan, L., BaAs, F., ET AL. (2008). Disrupted dopaminergic neurotransmission in 22q11 deletion syndrome. Neuropsychopharmacology, 33, 1252-1258.

Botvinick, M. M., Cohen, J. D., \& Carter, C. S. (2004). Conflict monitoring and anterior cingulate cortex: An update. Trends in Cognitive Sciences, 8, 539-546.

Bunge, S. A., Dudukovic, N. M., Thomason, M. E., Vaidya, C. J., \& Gabrieli, J. D. E. (2002). Immature frontal lobe contributions to cognitive control in children: Evidence from fMRI. Neuron, 33, 301311.

Chen, J., Lipska, B. K., Halim, N., Ma, Q. D., Matsumoto, M., MelHEM, S., ET AL. (2004). Functional analysis of genetic variation in catechol-O-methyltransferase (COMT): Effects on mRNA, protein, and enzyme activity in postmortem human brain. American Journal of Human Genetics, 75, 807-821.

Debbane, M., Schaer, M., Farhoumand, R., Glaser, B., \& Eliez, S. (2006). Hippocampal volume reduction in 22q11.2 deletion syndrome. Neuropsychologia, 44, 2360-2365.

Diamond, A., Briand, L., Fossella, J., \& Gehlbach, L. (2004). Genetic and neurochemical modulation of prefrontal cognitive functions in children. American Journal of Psychiatry, 161, 125-132.

Egan, M. F., Goldberg, T. E., Kolachana, B. S., Callicott, J. H., Mazzanti, C. M., Straub, R. E., ET al. (2001). Effect of COMT Val ${ }^{108 / 158}$ Met genotype on frontal lobe function and risk for schizophrenia. Proceedings of the National Academy of Sciences, 98, 6917-6922.

Eliez, S., Blasey, C. M., Schmitt, E. J., White, C. D., Hu, D., \& Reiss, A. L. (2001). Velocardiofacial syndrome: Are structural changes in the temporal and mesial temporal regions related to schizophrenia? American Journal of Psychiatry, 158, 447-453.

Fan, J., McCandliss, B. D., Sommer, T., Raz, A., \& Posner, M. I. (2002). Testing the efficiency and independence of attentional networks. Journal of Cognitive Neuroscience, 14, 340-347.

Freund, T. F., Powell, J. F., \& Smith, A. D. (1984). Tyrosine hydroxylase-immunoreactive boutons in synaptic contact with identified striatonigral neurons, with particular reference to dendritic spines. Neuroscience, 13, 1189-1215. 
Glaser, B., Debbane, M., Hinard, C., Morris, M. A., Dahoun, S. P., Antonarakis, S. E., \& Eliez, S. (2006). No evidence for an effect of COMT Val ${ }^{158}$ Met genotype on executive function in patients with 22q11 deletion syndrome. American Journal of Psychiatry, 163, 537-539.

Gothelf, D., Eliez, S., Thompson, T., Hinard, C., Penniman, L., FEInSTEIN, C., ET AL. (2005). COMT genotype predicts longitudinal cognitive decline and psychosis in 22q11.2 deletion syndrome. Nature Neuroscience, 8, 1500-1502.

Gothelf, D., Michaelovsky, E., Frisch, A., Zohar, A. H., PresBURGER, G., BURG, M., ET AL. (2007). Association of the low-activity COMT ${ }^{158}$ Met allele with ADHD and OCD in subjects with velocardiofacial syndrome. International Journal of Neuropsychopharmacology, 10, 301-308.

Grace, A. A., Floresco, S. B., Goto, Y., \& Lodge, D. J. (2007). Regulation of firing of dopaminergic neurons and control of goal-directed behaviors. Trends in Neurosciences, 30, 220-227.

Gratton, G., Coles, M. G., \& Donchin, E. (1992). Optimizing the use of information: Strategic control of activation of responses. Journal of Experimental Psychology: General, 121, 480-506.

Honey, G. D., Suckling, J., Zelaya, F., Long, C., Routledge, C., JACKSON, S., ET AL. (2003). Dopaminergic drug effects on physiological connectivity in a human cortico-striato-thalamic system. Brain, 126, 1767-1781.

Karoum, F., Chrapusta, S. J., \& Egan, M. F. (1994). 3-Methoxytyramine is the major metabolite of released dopamine in the rat frontal cortex: Reassessment of the effects of antipsychotics on the dynamics of dopamine release and metabolism in the frontal cortex, nucleus accumbens, and striatum by a simple two pool model. Journal of Neurochemistry, 63, 972-979.

Kates, W. R., Antshel, K. M., Abdulsabur, N., Colgan, D., Funke, B., Fremont, W., ET AL. (2006). A gender-moderated effect of a functional COMT polymorphism on prefrontal brain morphology and function in velo-cardio-facial syndrome (22q11.2 deletion syndrome). American Journal of Medical Genetics, 141B, 274-280.

Kimberg, D. Y., \& D'Esposito, M. (2003). Cognitive effects of the dopamine receptor agonist pergolide. Neuropsychologia, 41, 1020-1027.

Kornhuber, J., \& Kornhuber, M. E. (1983). Axo-axonic synapses in the rat striatum. European Neurology, 22, 433-436.

Maynard, T. M., Haskell, G. T., Lieberman, J. A., \& LaMantia, A. S. (2002). 22q11 DS: Genomic mechanisms and gene function in DiGeorge/velocardiofacial syndrome. International Journal of Developmental Neuroscience, 20, 407-419.

Mayr, U., Awh, E., \& Laurey, P. (2003). Conflict adaptation effects in the absence of executive control. Nature Neuroscience, 6, 450-452.
Murphy, K. C. (2002). Schizophrenia and velo-cardio-facial syndrome. Lancet, 359, 426-430.

Rosenberg, D. R., \& Lewis, D. A. (1995). Postnatal maturation of the dopaminergic innervation of monkey prefrontal and motor cortices: A tyrosine hydroxylase immunohistochemical analysis. Journal of Comparative Neurology, 358, 383-400.

SEAmans, J. K., \& YANG, C. R. (2004). The principal features and mechanisms of dopamine modulation in the prefrontal cortex. Progress in Neurobiology, 74, 1-58.

Shashi, V., Keshavan, M. S., Howard, T. D., Berry, M. N., BaseHORE, M. J., LEWANDOWSKI, E., ET AL. (2006). Cognitive correlates of a functional COMT polymorphism in children with 22q11.2 deletion syndrome. Clinical Genetics, 69, 234-238.

Simon, T. J., Bearden, C. E., McGinn, D. M., \& Zackai, E. (2005). Visuospatial and numerical cognitive deficits in children with chromosome 22q11.2 deletion syndrome. Cortex, 41, 145-155.

Sobin, C., Kiley-Brabeck, K., \& Karayiorgou, M. (2005). Lower prepulse inhibition in children with the $22 \mathrm{q} 11$ deletion syndrome. American Journal of Psychiatry, 162, 1090-1099.

Tunbridge, E. M., Weickert, C. S., Kleinman, J. E., Herman, M. M., Chen, J., Kolachana, B. S., Et Al. (2007). Catechol-omethyltransferase enzyme activity and protein expression in human prefrontal cortex across the postnatal lifespan. Cerebral Cortex, 17, $1206-1212$.

Wahlstrom, D., White, T., Hooper, C. J., VrsheK-Schallhorn, S., Oetting, W. S., Brott, M. J., \& Luciana, M. (2007). Variations in the catechol O-methyltransferase polymorphism and prefrontally guided behaviors in adolescents. Biological Psychiatry, 61, 626-632.

Williams, G. V., \& Goldman-RAKIC, P. S. (1995). Modulation of memory fields by dopamine D1 receptors in prefrontal cortex. Nature, 376, 572-575.

Winterer, G., Musso, F., Vucurevic, G., Stoeter, P., Konrad, A., SEKER, B., ET AL. (2006). COMT genotype predicts BOLD signal and noise characteristics in prefrontal circuits. NeuroImage, 32, 1722-1732.

Winterer, G., \& Weinberger, D. R. (2004). Genes, dopamine and cortical signal-to-noise ratio in schizophrenia. Trends in Neurosciences, 27, 683-690.

Zavitsanou, K., Cranney, J., \& Richardson, R. (1999). Dopamine antagonists in the orbital prefrontal cortex reduce prepulse inhibition of the acoustic startle reflex in the rat. Pharmacology, Biochemistry, \& Behavior, 63, 55-61.

(Manuscript received January 31, 2008; revision accepted for publication August 8, 2008.) 